\title{
Mechanical characterisation of nanocellulose composites after structural modification
}

\author{
H. Takagi ${ }^{1}$, A. N. Nakagaito ${ }^{1}$, K. Nishimura ${ }^{2} \&$ T. Matsui $^{2}$ \\ ${ }^{1}$ Graduate School of Science and Technology, \\ Tokushima University, Japan \\ ${ }^{2}$ Graduate School of Advanced Technology and Science, \\ Tokushima University, Japan
}

\begin{abstract}
Recently cellulose nanofiber has attracted researchers' attention as a new biobased reinforcing phase, because the mechanical property of the cellulose nanofiber seems to be excellent; for example, tensile strength and modulus are about $2 \mathrm{GPa}$ and about $140 \mathrm{GPa}$. However, the mechanical properties of polymer composites reinforced by the cellulose nanofiber are markedly lower than expected. In this study, polyvinyl alcohol-based nanocellulose fiber-reinforced composites were fabricated as a model nanocomposite system. We tried to improve the mechanical properties of the cellulose nanofiber-reinforced composites by controlling the orientation of cellulose nanofiber through multiple mechanical extension treatments. In the present study, the alignment of cellulose nanofiber in polymeric matrix can be controlled by applying 10 times mechanical extensions at most. The effectiveness of this fiber alignment control has been successfully demonstrated; the tensile strength and Young's modulus of the cellulose nanofiber-reinforced composites after the mechanical extension treatments reached almost the two-fold value as compared with those of the untreated composites.
\end{abstract}

Keywords: green composites, nanocomposites, cellulose nanofiber, fiber orientation, mechanical properties.

\section{Introduction}

Natural fiber-reinforced biodegradable composites (namely green composites) have gotten a lot of scientists' attention in the research field of composite 
materials, because the green composites have various advantages; such as low density, high strength, high modulus, and biodegradable nature $[1,2]$. Thus, the green composites are thought to be a possible alternative composite material for traditional petroleum-derived polymer composites with high environmental loads. Many studies concerned tensile and flexural properties of the green composites reinforced by a wide variety of thick natural fibers, for example jute, ramie, hemp, and bamboo fibers [3-5].

As potential nanoscale reinforcement derived from biomass, cellulose nanofibers have drawn researchers' attention. The pure cellulose nanofibers, which contain neither lignin nor hemicellulose, typically have diameter of 5-100 $\mathrm{nm}$. It is thought that the cellulose nanofibers possess excellent mechanical properties, e.g., tensile strength of about $1.7 \mathrm{GPa}[6]$ and Young's modulus of about $140 \mathrm{GPa}$ [7]. These excellent mechanical properties are comparable with those of glass and aramid fibers.

The mechanical properties reported for the cellulose nanofiber-reinforced polymer composites are much lower than expected. The starch-based green composites reinforced by cellulose nanofiber have flexural strength of about 68 $\mathrm{MPa}$ and flexural modulus of $6.6 \mathrm{GPa}$ [8]. There are several reasons for this inferior mechanical property; one of highly possible reasons is a fiber alignment, i.e. random orientation of cellulose nanofiber in the polymer matrix.

In the other nanocomposite system, as a possible orientation control method for the nanofiber, magnetic field orientation control and electric field orientation control of carbon-based nanofillers were studied until now. In the research using the electric field orientation control, the fiber orientation of carbon nanotube was performed by applying electric field, and then fabricated epoxy-based polymer composite [9]. It was reported that the modulus in the normal direction of the orientation-controlled nanocomposites was lower than that of randomly oriented composites. Additionally, there was report on orientation control for cellulose whisker by applying an electric field [10].

In this study we tried to control the cellulose nanofiber orientation by multiple mechanical extension method. In the multiple mechanical extension method, the alignment of cellulose nanofiber in polymeric matrix can be controlled by applying 2-10 times mechanical extensions. The effectiveness of the fiber alignment control has been successfully demonstrated experimentally; the tensile properties of the cellulose nanofiber composites after fiber alignment treatment have almost the two-fold values as compared with those of the untreated composites.

\section{Experimental methods}

\subsection{Preparation of nanocomposites}

Polyvinyl alcohol powder (162-16325, Wako Pure Chemical Industries Co., Ltd. Japan, hereafter PVA) was used as a biodegradable polymer matrix. Commercially available cellulose nanofiber (KY-100G, Co., Ltd. Daicel Co., Japan, hereafter $\mathrm{CNF}$ ) was used as reinforcement. PVA powder was dissolved in hot water using a mantle heater at $80^{\circ} \mathrm{C}$, after $25 \mathrm{~g}$ of PVA was mixed with distilled water of $475 \mathrm{~g}$, and $5 \mathrm{wt} . \%$ PVA solution was prepared. After that, CNF of $1.5 \mathrm{~g}$ was mixed with distilled water of $135 \mathrm{~g}$ to prepare water suspension with $1 \mathrm{wt} . \% \mathrm{CNF}$. This 
suspension and the PVA solution were mixed to make the PVA mixture having CNF content of $30 \mathrm{wt} . \%$. Afterwards, the composite material was produced by pouring this $\mathrm{PVA} / \mathrm{CNF}$ suspension in a plastic container (i.e. casting), and then dried it in an oven kept at $30^{\circ} \mathrm{C}$, resulting in a final nanocomposites sheet.

\subsection{Mechanical extension treatment for nanocomposites}

The PVA/cellulose nanofiber-reinforced composites (CNF content $=30 \mathrm{wt}$. $\%$ ) were cut into rectangular shape $(50 \times 120 \mathrm{~mm})$, and then immersed in distilled water for $10 \mathrm{~min}$. The water-soaked composite sample was mechanically extended by a hand-made tension instrument as shown in Fig. 1, and then dried for $24 \mathrm{~h}$. These steps were repeated 10 times in the same manner. Finally, the composite sample was hot-pressed at $15 \mathrm{MPa}$ and at $180^{\circ} \mathrm{C}$ for $10 \mathrm{~min}$.

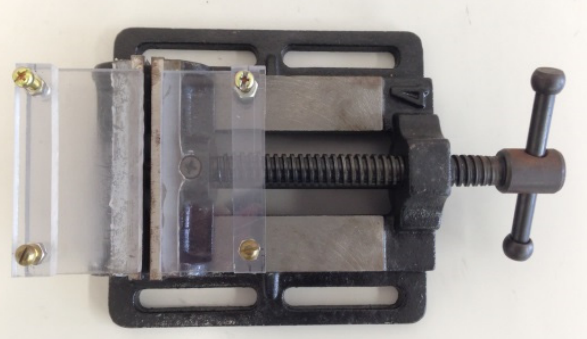

Figure 1: Device for mechanical extension.

\subsection{Tensile testing}

Tensile tests of all the samples were carried out at a tensile speed of $1.0 \mathrm{~mm} / \mathrm{min}$ on an Instron universal testing machine (Model 5567, U.S.A.). The shape of the tensile test specimen was $10 \mathrm{~mm}$ wide, $90 \mathrm{~mm}$ long, and the gauge length was 30 $\mathrm{mm}$. Tensile strength and Young's modulus were determined from the measured stress-strain curves.

\section{Results and discussion}

\subsection{Effect of number of extension treatment}

The variation in tensile strength and Young's modulus as a function of the number of mechanical extension treatments is indicated in Fig. 2, showing the effect of mechanical extension treatments on the mechanical properties of PVA/CNF nanocomposites. It can be seen that the nanocomposite mechanically extended just once have higher mechanical properties than original nanocomposites. The 
mechanical properties of nanocomposites peak at 5 times extension; however, they decrease at 10 times extensions.

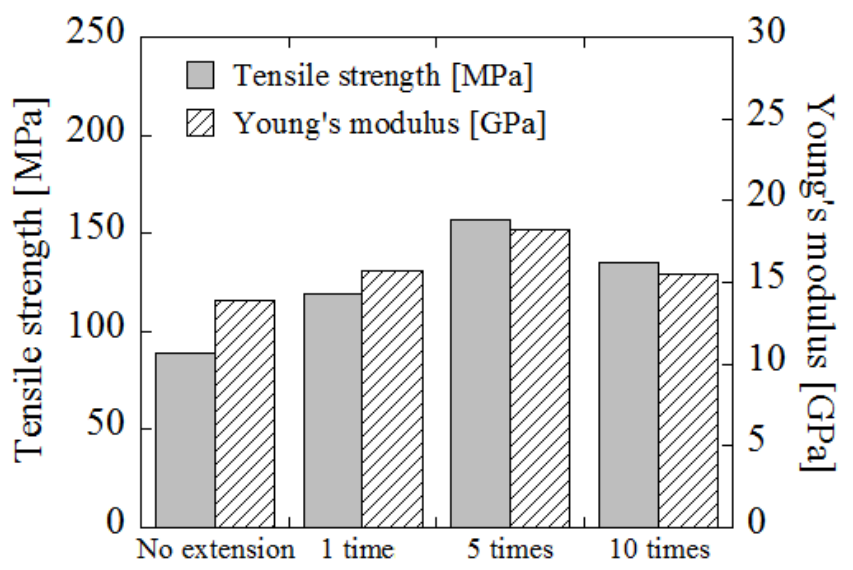

Figure 2: Variation of tensile strength and Young's modulus of PVA/CNF nanocomposites; fiber content $=30 \mathrm{wt} . \%$.

The corresponding stress-strain curves of the nanocomposites are shown in Fig. 3. We can cee that not only strength and modulus but also deformability is improved after mechanical extension treatments. In addition, this higher tensile strength might be attributed to the effect of the proper fiber alignment in polymer matrix.

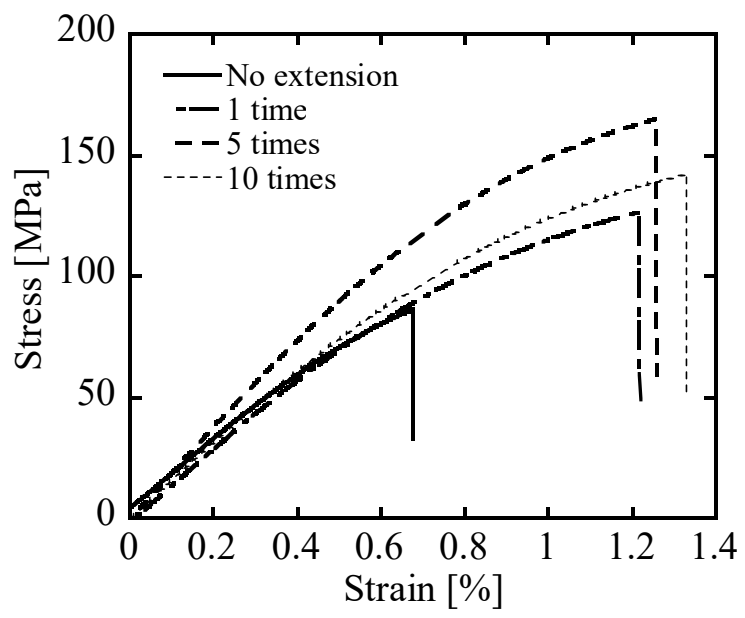

Figure 3: Typical stress-strain curves of PVA/CNF nanocomposites; fiber content $=30 \mathrm{wt} . \%$. 


\subsection{Effect of testing direction}

In order to check the effectiveness of multiple mechanical extension treatment, we have evaluated the effect of pulling direction during tensile testing on the mechanical properties of PVA/CNF nanocomposites. Figure 4 show the variation in tensile strength and Young's modulus of PVA/CNF nanocomposites tensiletested along 0 degree and 90 degrees directions (Fig. 5). The corresponding date

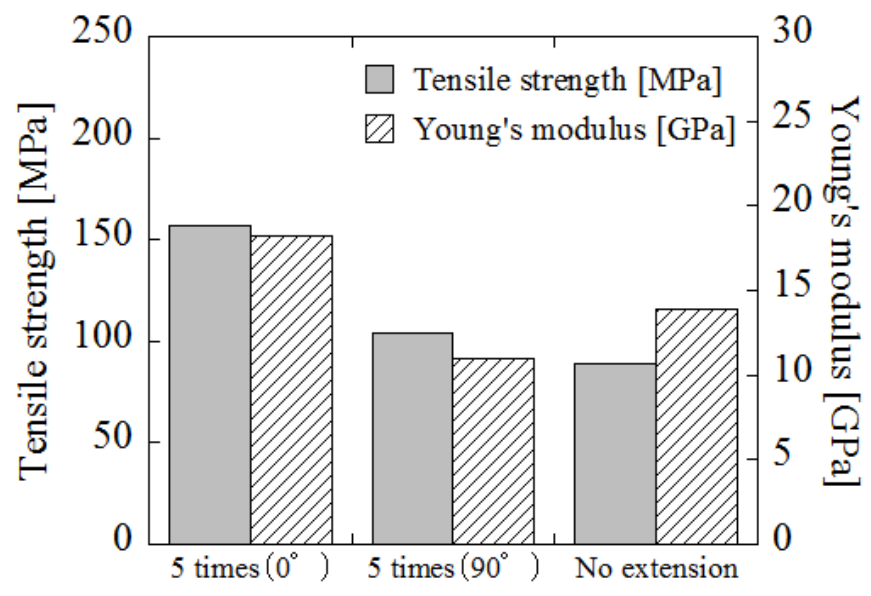

Figure 4: Tensile strength and Young's modulus of PVA/CNF nanocomposites; fiber content $=30 \mathrm{wt} . \%$.

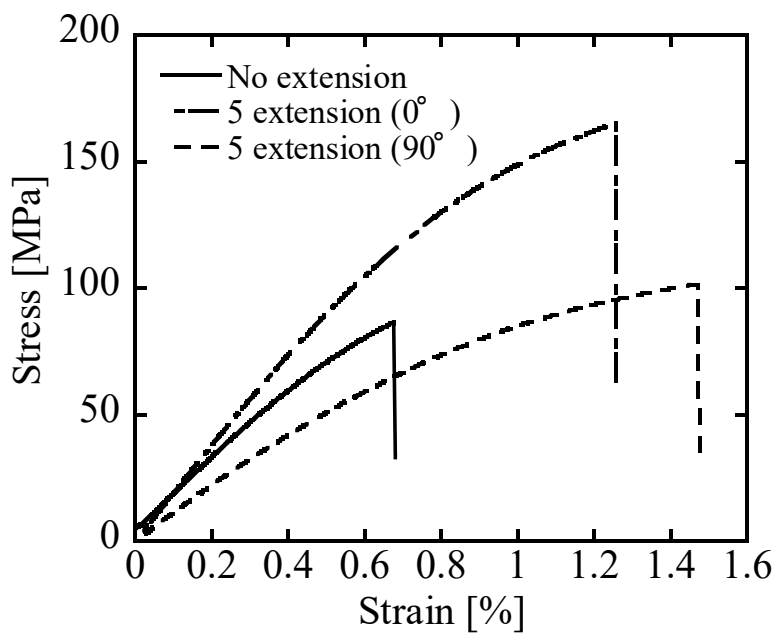

Figure 5: Typical stress-strain curves of PVA/CNF nanocomposites tested along 0 and 90 degrees; fiber content $=30 \mathrm{wt} . \%$. 
for non-treated nanocomposites is also indicated in the same figure for reference. The mechanical properties of the composites tested along 90 degrees direction are lower than those of untreated nanocomposites, indicating that the effectiveness of multiple mechanical extension treatment.

\section{Conclusions}

The effect of multiple mechanical extension treatment on the mechanical property of PVA/CNF nanocomposites was investigated. From the results obtained in these investigations, the following conclusions were reached.

1) Both tensile strength and Young's modulus peaked after 5 times extension treatment. However too many extension treatments (e.g. 10 times) do not contribute to the mechanical properties of nanocomposites.

2) The tensile strength of nanocomposites tensile-tested along 90 degrees, namely along the transverse direction, was lower than that of untreated nanocomposites. This result also supports the effectiveness of the multiple mechanical extension treatment.

\section{Acknowledgement}

This work was supported by Japan Society for the Promotion of Science (JSPS) KAKENHI Grant Number 15K14148.

\section{References}

[1] Netravali, A. N. \& Chabba, S., Composites get greener. Materials Today, 6(4), pp. 22-29, 2003.

[2] Pandey, J. K., Takagi, H., Nakagaito, A. N., Saini, D. R. \& Ahn, S. H., An overview on the cellulose based conducting composites. Composites Part B: Engineering, 43(7), pp. 2822-2826, 2012.

[3] Lodha, P. \& Netravali, A. N., Characterization of interfacial and mechanical properties of "green" composites with soy protein isolate and ramie fibre. Journal of Materials Science, 37(17), pp. 3657-3665, 2002.

[4] Takagi, H., Liu, K., Osugi, R., Nakagaito, A. N. \& Yang, Z., Heat barrier properties of green composites. Journal of Biobased Materials and Bioenergy, 6(4), pp. 470-474, 2012.

[5] Liu, K., Takagi, H., Osugi, R. \& Yang, Z., Effect of physicochemical structure of natural fiber on transverse thermal conductivity of unidirectional abaca/bamboo fiber composites, Composites Part A: Applied Science and Manufacturing, 43(8), pp. 1234-1241, 2012.

[6] Sakurada, I., Nukushima, Y. \& Ito, T., Experimental determination of the elastic modulus of crystalline regions in oriented polymers. Journal of Polymer Science, 57(165), pp. 651-660, 1962.

[7] Page, D. H. \& EL-Hosseiny, F., The mechanical properties of single wood pulp fibres, Part VI Fibril angle and the shape of the of the stress-strain curve. Journal of Pulp and Paper Science, 9(4), pp. 99-100, 1983. 
[8] Takagi, H. \& Asano, A., Effects of processing conditions on flexural properties of cellulose nanofiber reinforced "green" composites. Composites Part A: Applied Science and Manufacturing, 39(4), pp. 685689, 2008.

[9] Hilding, J. M., Hong, M. P. L. \& Grulke, E. A., Alignment of dispersed multiwalled carbon nanotubes in low strength AC electrical fields. Journal of Nanoscience and Nanotechnology, 5(5), pp. 742-746, 2005.

[10] Kvien, I. \& Oksman, K., Orientation of cellulose nanowhiskers in polyvinyl alcohol. Applied Physics A-Materials Science \& Processing, 87(4), pp. 641-643, 2007. 